\title{
New insights into the Be/X-ray binary system MXB 0656-072 ${ }^{\star}$
}

\author{
E. Nespoli ${ }^{1,2}$, P. Reig ${ }^{3,4}$, and A. Zezas ${ }^{4}$ \\ ${ }^{1}$ Observatorio Astronómico de la Universidad de Valencia, Calle Catedrático Jose Beltran, 2, 46980 Paterna, Valencia, Spain \\ ${ }^{2}$ European Space Astronomy Centre (ESA/ESAC), Science Operations Department, Villanueva de la Cañada (Madrid), Spain \\ e-mail: elisa.nespoli@uv.es \\ 3 Foundation for Research and Technology - Hellas, IESL, Voutes, 71110 Heraklion, Crete, Greece \\ ${ }^{4}$ Physics Department, University of Crete, 71003 Heraklion, Crete, Greece
}

Received 11 May 2012 / Accepted 22 August 2012

\begin{abstract}
Context. The X-ray transient MXB 0656-072 is a poorly studied member of high-mass X-ray binaries. Based on the transient nature of the X-ray emission, the detection of pulsations, and the early-type companion, it has been classified as a Be X-ray binary (Be/XRB). However, the flaring activity covering a large fraction of a giant outburst is somehow peculiar.

Aims. Our goal is to investigate the multiwavelength variability of the high-mass X-ray binary MXB 0656-072.

Methods. We carried out optical spectroscopy and analysed all RXTE archive data, performing a detailed X-ray-colour, spectral, and timing analysis of both normal (type-I) and giant (type-II) outbursts from MXB 0656-072.

Results. This is the first detailed analysis of the optical counterpart in the classification region (4000-5000 A). From the strength and ratio of the elements and ions, we derive an $09.5 \mathrm{Ve}$ spectral type, in agreement with previous classification. This confirms its $\mathrm{Be}$ nature. The characterisation of the Be/XRB system relies on Balmer lines in emission in the optical spectra, long-term X-ray variability, and the orbital period vs. spin period and $\mathrm{EW}(\mathrm{H} \alpha)$ relation. The peculiar feature that distinguishes the type-II outburst is flaring activity, which occurs during the whole outburst peak, before a smoother decay. We interpret it in terms of magnetohydrodynamic instability. Colour and spectral analysis reveal a hardening of the spectrum as the flux increases. We explored the aperiodic X-ray variability of the system for the first time, finding a correlation of the central frequency and rms of the main timing component with luminosity, which extends up to a "saturation" flux of $1 \times 10^{-8} \mathrm{erg} \mathrm{cm}^{-2} \mathrm{~s}^{-1}$. A correlation between timing and spectral parameters was also found, pointing to an interconnection between the two physical regions responsible for both phenomenologies.
\end{abstract}

Key words. binaries: general - stars: emission-line, Be - stars: neutron

\section{Introduction}

$\mathrm{Be} / \mathrm{X}$-ray binaries (Be/XRBs) constitute a sub-class of highmass X-ray binaries (HMXBs) in which the companion is a Be star, i.e. a non-supergiant fast-rotating OB-star that during its life has shown at some point spectral lines in emission (see Reig 2011, for a recent review). They are also characterised by infrared excess, which means that they are brighter in the IR than their non-emitting counterparts of the same spectral type. Both phenomena, emission lines and IR excess, are thought to arise from a common cause, namely the presence of an extended circumstellar envelope around the stellar equator, made up of ionised gas that is expelled from the star in a way that is not yet completely understood. This complex scenario is referred to as the Be phenomenon (Porter \& Rivinius 2003; Ekström et al. 2008).

When a Be star is part of an X-ray binary, the system is usually transient, and the compact object is virtually always a pulsar, with typical spin periods ranging between $1-10^{3} \mathrm{~s}$. Be/XRBs are characterised by high variability on a wide range of both time scales (from seconds to years) and wavelengths, although the fastest variability is observed in the X-ray band. For longer periods, the variability is apparent in both high-energy and lowenergy wavelengths, and is attributed to major changes in the circumstellar disc structure. The complexity of the dynamics of

\footnotetext{
* The spectra are only available at the CDS via anonymous ftp to cdsarc.u-strasbg.fr $(130.79 .128 .5)$ or via

http://cdsarc.u-strasbg.fr/viz-bin/qcat?]/A+A/547/A103
}

the Be phenomenon and its relation with the accretion onto the compact object clearly require a multiwavelength approach in the study of these systems.

Even if its phenomenology is entangled and multi-faceted, the long-term X-ray variability in Be/XRBs is traditionally described by a classification into two types of outbursts. Type-I (or normal) outbursts are periodic or quasi-periodic events, occurring in correspondence (or close) to the periastron passage of the neutron star. They are generally short, with a typical duration of 0.2-0.3 $P_{\text {orb }}$, and show luminosities $L_{\mathrm{X}}=10^{36}-10^{37} \mathrm{erg} \mathrm{s}^{-1}$. Type-II (or giant) outbursts are unpredictable, long (one or more orbital periods), and bright events, with typical X-ray luminosities of $L_{\mathrm{X}}=10^{37}-10^{38} \mathrm{erg} \mathrm{s}^{-1}$, corresponding to up to the Eddington luminosity for a neutron star. The presence of quasiperiodic oscillations (QPOs) in some systems would support the suggestion of the formation of an accretion disc around the neutron star during type-II outbursts (see for instance Motch et al. 1991; Hayasaki \& Okazaki 2004).

The transient X-ray binary MXB 0656-072 was discovered by $S A S-3$ in September 1975, when a flux density of $80 \mathrm{mCrab}$ was reported (Clark et al. 1975), and subsequently observed twice in 1976 by Ariel V at 50 (March 19) and 70 (March 27) mCrab, respectively (Kaluzienski 1976). These intensities would convert into an X-ray luminosity of $\sim 2-3 \times 10^{36} \mathrm{erg} \mathrm{s}^{-1}$, assuming a distance of $3.9 \mathrm{kpc}$ (McBride et al. 2006). Therefore, they would correspond to the typical $\mathrm{X}$-ray luminosity range for type-I outbursts. 
Table 1. Journal of RXTE observations.

\begin{tabular}{lccc}
\hline \hline $\begin{array}{l}\text { N. of } \\
\text { pointings }\end{array}$ & $\begin{array}{c}\text { Proposal } \\
\text { ID }\end{array}$ & $\begin{array}{c}\text { MJD } \\
\text { range }\end{array}$ & $\begin{array}{c}\text { On-source } \\
\text { time (ks) }\end{array}$ \\
\hline 28 & 80067 & $52931.8-52975.4$ & 91.1 \\
33 & 80430 & $52966.9-53033.3$ & 38.6 \\
44 & 93032 & $54419.5-54748.6$ & 179.6 \\
123 & 93423 & $54449.1-54776.6$ & 189.1 \\
\hline
\end{tabular}

Although the discovery of the source dates back to more than 35 years ago, very little is known about the system. MXB 0656072 was only catalogued as an HMXB in 2003 after extended re-brightening, when its optical counterpart was identified and classified as an O9.7Ve star (Pakull et al. 2003), and a pulsed period of $160.7 \mathrm{~s}$ detected (Morgan et al. 2003). The energy spectrum of the source showed a cyclotron resonant energy feature (CRSF) at a central energy of $\sim 33 \mathrm{keV}$ (Heindl et al. 2003). This event was classified as a type-II outburst.

McBride et al. (2006) monitored the source over the 2003 major outburst and studied the average X-ray spectrum during the peak of the outburst and the change in the spin period. They did a pulse phase-resolved analysis and found that the width of the CRSF varied with pulse phase, being wider during the pulse decline. However, the energy of the CRSF did not change with pulse phase. A cyclotron line at $\sim 33 \mathrm{keV}$ implies a magnetic field strength of $3.7 \times 10^{12} \mathrm{G}$. They also present a timing analysis of the pulsations, including pulse profile dependence on energy and the changes in the spin period throughout the outburst. Recently, Yan et al. (2012) have presented a correlated optical/X-ray analysis of the system during type-I outbursts, after discovering a 101.2 days orbital period.

In this paper we analyse all RXTE data available for MXB 0656-072, which include the major 2003 outburst and a series of type-I outbursts observed in 2007-2008. We performed $\mathrm{X}$-ray colour, spectral, and timing analysis of the giant outburst, and colour and spectral analysis of the normal outbursts. We focus on the aperiodic variability and the study of the broad-band noise. Moreover, we present here additional optical spectroscopy that allows us to refine and robustly justify the spectral classification on one side, besides providing a long-term correlated $\mathrm{H} \alpha / \mathrm{X}$-ray follow up of the system.

\section{RXTE observations and data reduction}

RXTE followed the source during the first giant outburst observed since its discovery, starting in October 2003 for approximately three and a half months (MJD 52931-53033). Renewed activity of the source was detected by the RXTE in November 2007, lasting for one year (MJD 54 419-54 776), with luminosities lower than in 2003. The 2007 X-ray variability consisted of a series of four (quasi-)periodic flares, which are reminiscent of type-I outbursts. Table 1 shows the observation log. The total net exposure amounted to $129.7 \mathrm{ks}$ for the first event analysed here, and to $368.7 \mathrm{ks}$ for the second one.

We employed data from all the three instruments onboard RXTE (Bradt et al. 1993), the All-Sky Monitor (ASM), the Proportional Counter Array (PCA), and the High Energy X-ray Timing Experiment (HEXTE). The ASM incorporates three wide-angle shadow cameras equipped with proportional counters with a total collecting area of $90 \mathrm{~cm}^{2}$. It works in the 2$10 \mathrm{keV}$ energy range, mapping the $80 \%$ of the sky every $90 \mathrm{~min}$. The PCA consists of five proportional counter units (PCUs) with a total collecting area of $\sim 6250 \mathrm{~cm}^{2}$ and operates in the 2$60 \mathrm{keV}$ range, with a nominal energy resolution of $18 \%$ at $6 \mathrm{keV}$. The HEXTE comprises two clusters of four NaI/CsI scintillation counters, with a total collecting area of $2 \times 800 \mathrm{~cm}^{2}$, sensitive in the $15-250 \mathrm{keV}$ band with a nominal energy resolution of $15 \%$ at $60 \mathrm{keV}$. Both the PCA and the HEXTE have a maximum time resolution of $\sim 1 \mu \mathrm{s}$.

Data reduction was performed using HEASOFT version 6.9. An energy spectrum was obtained for each pointing, after filtering out unsuitable data according to the recommended criteria ${ }^{1}$, employing Standard 2 mode data from the PCA (PCU2 only) and Standard (archive) mode from the HEXTE Cluster A, with a time resolution of $16 \mathrm{~s}$. The PCA and HEXTE spectra were extracted, background-subtracted, and dead-time corrected. For the PCA, the 3-30 keV energy range was retained, while the HEXTE provided a partially overlapping extension from 25 to $100 \mathrm{keV}$. In the case of the 2003 outburst, for each observation, the two resulting spectra were simultaneously fitted with XSPEC v. 12.6.0 (Arnaud 1996). For the 2007-2008 event, three average PCA spectra were extracted in three luminosity ranges $\left(<1 \times 10^{-9}, 1-\right.$ $2.5 \times 10^{-9}$, and $>2.5 \times 10^{-9} \mathrm{erg} \mathrm{cm}^{-2} \mathrm{~s}^{-1}$ respectively) and fitted between 3-60 keV in order to constrain the CRSF. Then a spectrum for each pointing was fitted between $3-30 \mathrm{keV}$, fixing the CRSF parameters to the corresponding average ones. HEXTE 2007-2008 spectra were too faint to be employed. During the fitting, a systematic error of $0.6 \%$ was added to the PCA spectra.

Power spectral density (PSD) was computed using PCA Event or Single_Bit data. We first extracted, for each observation, a light curve in the energy range $\sim 3.5-17 \mathrm{keV}$ (channels 839 ) with a time resolution of $2^{-6}$ s. The light curve was then divided into 128-s segments, and a fast Fourier transform was computed for each segment. The final PSD was computed as the average of all the power spectra obtained for each segment. These averaged power spectra were logarithmically rebinned in frequency and corrected for dead time effects according to the prescriptions given in Nowak et al. (1999). Power spectra were normalised such that the integral over the PSD is equal to the squared fractional rms amplitude, according to the so-called rms-normalisation (Belloni \& Hasinger 1990; Miyamoto et al. 1991).

\section{Optical observations}

Optical spectroscopic observations of the companion star to MXB 0656-072 were performed using the Fred Lawrence Whipple Observatory at Mt. Hopkins (Arizona, USA) and the 1.3-m telescope from the Skinakas observatory (Crete, Greece). Table 2 gives the log of the observations.

The $1.3 \mathrm{~m}$ telescope of the Skinakas Observatory (SKO) was equipped with a $2000 \times 800$ ISA SITe CCD and a $13021 \mathrm{~mm}^{-1}$ grating (on 30 September 2010) and $24001 \mathrm{~mm}^{-1}$ (on 8 November 2011), giving a nominal dispersion of $\sim 1 \AA /$ pixel and $\sim 0.5 \AA /$ pixel, respectively. We also observed MXB 0656072 in queue mode with the 1.5-m telescope (FLWO) at Mt. Hopkins (Arizona) and the FAST-II spectrograph plus FAST3 CCD, a back-side illuminated $2688 \times 512$ UA STA520A chip with $15 \mu \mathrm{m}$ pixels. Spectra of comparison lamps were taken before each exposure to account for small variations in the wavelength calibration during the night. To ensure a homogeneous

\footnotetext{
1 Among which, elevation from the Earth greater than $10^{\circ}$ and pointing offset lower than $0.02^{\circ}$; see PCA digest at http://heasarc.gsfc.nasa.gov/docs/xte/pca_news.html
} 
Table 2. Log of the optical observations.

\begin{tabular}{lcccccc}
\hline \hline Date & $\begin{array}{c}\text { JD } \\
(2400000+)\end{array}$ & Telescope & $\begin{array}{c}\text { Grating } \\
(1 / \mathrm{mm})\end{array}$ & $\begin{array}{c}\text { Wavelength } \\
\text { range }(\AA)\end{array}$ & $\begin{array}{c}E W(\mathrm{H} \alpha) \\
(\AA)\end{array}$ & $\begin{array}{c}E W(\mathrm{H} \beta) \\
(\AA)\end{array}$ \\
\hline $14-11-2009^{*}$ & 55150.41 & FLWO & 600 & $4760-6760$ & $-18.4 \pm 1.0$ & $-2.98 \pm 0.11$ \\
$12-01-2010$ & 55209.37 & FLWO & 600 & $4730-6730$ & $-20.9 \pm 1.5$ & $-3.43 \pm 0.08$ \\
$16-01-2010$ & 55213.21 & FLWO & 600 & $4740-6740$ & $-21.2 \pm 0.8$ & $-3.60 \pm 0.09$ \\
$30-09-2010$ & 55470.60 & SKO & 1301 & $5300-7300$ & $-10.8 \pm 0.4$ & - \\
$30-10-2010$ & 55501.01 & FLWO & 1200 & $6200-7200$ & $-11.9 \pm 0.6$ & - \\
$29-11-2010$ & 55530.83 & FLWO & 1200 & $6200-7200$ & $-11.6 \pm 0.6$ & - \\
$03-10-2011$ & 55838.98 & FLWO & 1200 & $6200-7200$ & $-12.2 \pm 0.7$ & - \\
$01-11-2011$ & 55867.88 & FLWO & 1200 & $6200-7200$ & $-13.0 \pm 0.7$ & - \\
$08-11-2011$ & 55873.60 & SKO & 2400 & $3940-5040$ & - & $-2.23 \pm 0.07$ \\
$23-11-2011$ & 55889.93 & FLWO & 1200 & $6200-7200$ & $-14.1 \pm 0.8$ & - \\
$31-11-2011^{*}$ & 55927.71 & FLWO & 1200 & $6200-7200$ & $-15.4 \pm 0.8$ & - \\
$19-01-2012$ & 55946.79 & FLWO & 1200 & $6200-7200$ & $-15.3 \pm 0.8$ & - \\
$22-01-2012^{*}$ & 55927.71 & FLWO & 1200 & $6200-7200$ & $-15.2 \pm 0.8$ & - \\
\hline
\end{tabular}

Notes. ${ }^{(*)}$ Average of two measurements.

processing of the spectra, all of them were normalised with respect to the local continuum, which was rectified to unity by employing a spline fit.

We measured calibrated photometry of the optical counterpart with dedicated observations for the first time, performed from the 1.3-m telescope of the Skinakas Observatory on 2 November 2010 (JD 2455 503.6). MXB 0656-072 was observed through the Johnson $B, V, R$, and $I$ filters. The telescope was equipped with a $2048 \times 2048$ ANDOR CCD with a $13.5 \mu \mathrm{m}$ pixel size. Standard stars from the Landolt list (Landolt 2009) were used for the transformation equations. Reduction of the data was carried out in the standard way using the IRAF tools for aperture photometry. The resulting magnitudes are: $B=$ $13.25 \pm 0.02 \mathrm{mag}, V=12.25 \pm 0.02 \mathrm{mag}, R=11.63 \pm 0.02 \mathrm{mag}$, and $I=10.97 \pm 0.02 \mathrm{mag}$.

\section{Spectral class}

The only report of the spectral type of the massive companion in MXB 0656-072 is given by Pakull et al. (2003), who suggest an O9.7V spectral class. Blue- and red-end optical spectra covering the period 2005-2009 are also presented in Yan et al. (2012).

Figure 1 shows the optical spectrum of MXB 0656-072 in the region 4000-4800 $\AA$ from the Skinakas observatory. This spectrum is the average of three spectra obtained with a total exposure time of $7200 \mathrm{~s}$ each. The distinct presence of He II lines indicates an O-type star, while the presence of He I lines implies that the spectral type must be later than O8. Some He I lines, such as $\lambda 4713$ and $\lambda 4921$, appear (partially) in emission and cannot be separated out from the continuum. The ratio C III $\lambda 4650$ to He II $\lambda 4686$ is close to 1 , which agrees with an O9-O9.5 type (Walborn \& Fitzpatrick 1990). The ratio He II $\lambda 4200 / H e$ I $\lambda 4144$ allows us to distinguish between these two close sub-classes (Walborn 1971). This ratio is approximately 1 in O9 stars and lower than 1 in O9.5 stars. In MXB 0656-072 this ratio appears to be slightly lower than 1 , favouring the later type classification. Also, since the strength of the He I $\lambda 4144$ might be diminished by the emission from the circumstellar disc, the 09.5 class appears to be more likely. On the other hand, given the relatively low $\mathrm{S} / \mathrm{N}$ in this part of the spectrum and the uncertainty introduced in the definition of the continuum during the normalisation, an $\mathrm{O} 9$ spectral type cannot be completely ruled out.

As for the luminosity class, the strong He II $\lambda 4686$ absorption accompanied by weak N III $\lambda 4634-4640-4642$ clearly

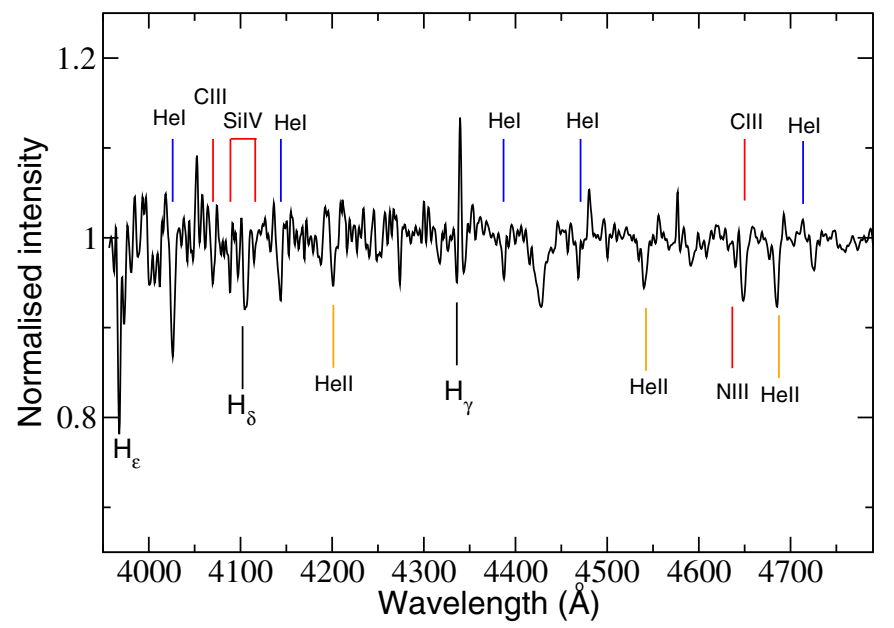

Fig. 1. The optical spectrum of the optical counterpart to MXB 0656072 in the $4000-4800 \AA$ region. The identified lines correspond to He II $\lambda 4200, \lambda 4541, \lambda 4686$, He I $\lambda 4026, \lambda 4144, \lambda 4387, \lambda 4471, \lambda 4713$, C III $\lambda 4070, \lambda 4650, N$ III $\lambda 4640$, Si IV $\lambda 4089, \lambda 4116$, and the hydrogen lines of the Balmer series between $\mathrm{H}_{\beta}$ and $\mathrm{H}_{\epsilon}$. A Gaussian smoothing filter $(\sigma=1)$ was applied to reduce the noise.

indicates a main-sequence star, and so does the strength of Si IV $\lambda 4089$ in comparison with that of He I $\lambda 4144$. We conclude that the optical counterpart to MXB 0656-072 is an $09.5 \mathrm{~V}$ $\operatorname{star}^{2}$.

In addition to He I lines, the Balmer series of hydrogen lines are strongly affected by emission. Even $\mathrm{H} \epsilon$ appears to be filledin with emission. Columns 6 and 7 in Table 2 give the equivalent width of the $\mathrm{H} \alpha$ and $\mathrm{H} \beta$ lines. A long-term decrease in the strength of these two lines is observed.

\section{Results}

We analysed all the observations of MXB 0656-072 in the RXTE archive. We separated the observations into two intervals

2 The interpolated class O9.7 suggested by Pakull et al. (2003) is mainly used for supergiants. The primary defining criterion for the O9.7 type is He II $\lambda 4541 \approx \mathrm{Si}$ III $\lambda 4552$ (Walborn \& Fitzpatrick 1990). According to Walborn (1971), it does not appear that this subdivision is useful at the lower luminosities (class II and below) because the $\mathrm{Si}$ III lines are too weak. 

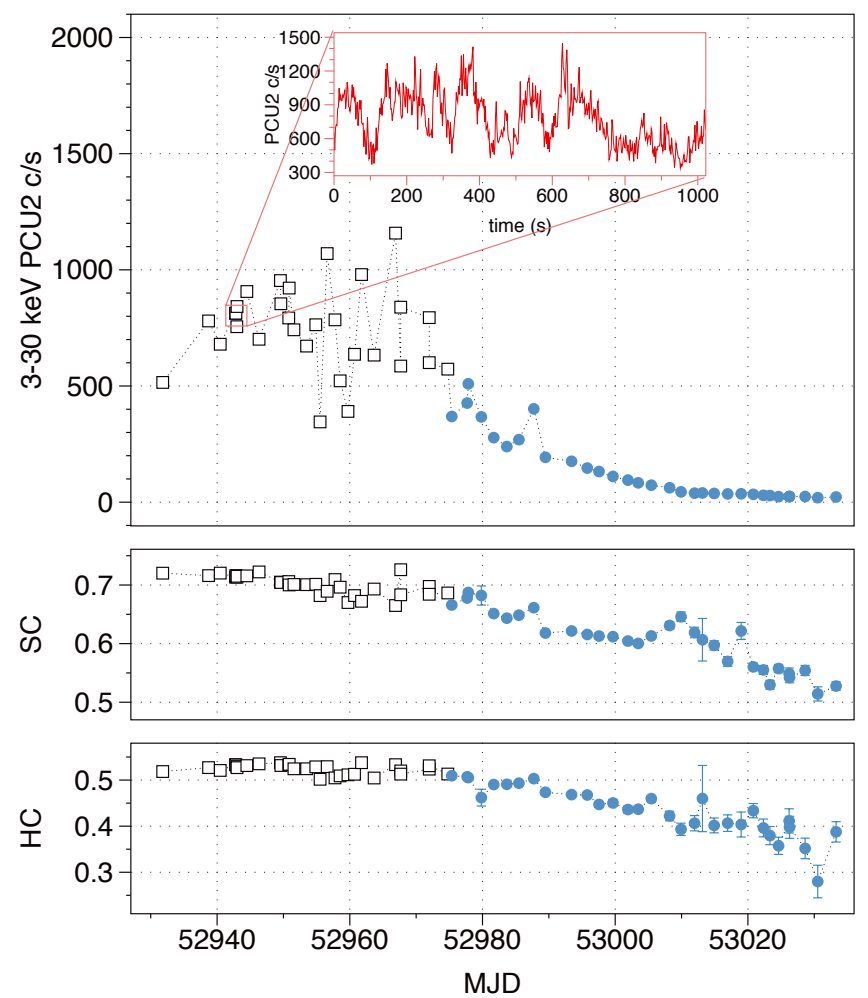

Fig. 2. PCA light curve and colour behaviour during the 2003 outburst. In the inset, $2 \mathrm{~s}$ resolution light curve for one pointing during the flaring phase.

corresponding to two significant events. The first interval started on 20 October 2003 (MJD 52932) and covered a total of 101.5 days. This interval includes a giant (type II) outburst. Assuming a distance of $3.9 \mathrm{kpc}$ (McBride et al. 2006), the maximum 3-30 keV luminosity during the outburst was $L_{X}=3.7 \times$ $10^{37} \mathrm{erg} \mathrm{s}^{-1}$, registered at MJD $\sim 52966.9$. The second interval covered the period 27 November 2007 to 11 November 2008 (MJD 54 419.5-54776.6) and includes a series of minor (type I) outbursts. The peak X-ray luminosity of these outbursts was $L_{\mathrm{X}}=1.37 \times 10^{37} \mathrm{erg} \mathrm{s}^{-1}$.

\subsection{Type II outburst}

\subsubsection{Colour analysis}

The PCA light curve and colour behaviour during the 2003 outburst is presented in Fig. 2. The outburst showed strong flaring behaviour during the peak phase, followed by smoother decay. Each point corresponds to an RXTE pointing and is directly obtained from PCU2 count rate. Different symbols mark the different outburst phases, the flare-like phase (open squares), and the smoother decay (filled circles). Error bars are the same size as the points whenever they do not appear in the plots. The X-ray colours were defined as follows, soft colour (SC): 7$10 \mathrm{keV} / 4-7 \mathrm{keV}$; hard colour (HC): 15-30 keV/10-15 keV. The two colours follow identical patterns during the outburst, both correlating with flux. In the inset, a zoomed-in view is shown, presenting the light curve for one PCA pointing, with a $2 \mathrm{~s}$ time resolution. From the inset it is clear that the flare-like activity displayed during the outburst is large-scale behaviour that in fact corresponds to variability on various time scales, if investigated at higher time resolution: besides the $\sim 160$ s pulse period, slower

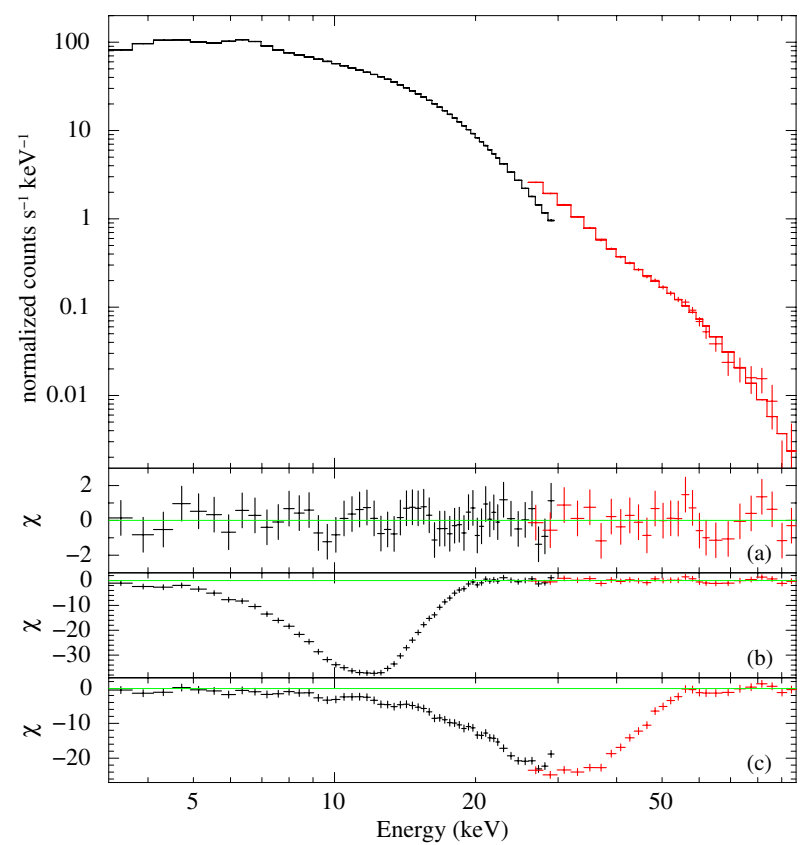

Fig. 3. Typical spectrum for an observation during the flaring phase of the giant outburst (obsid: 80067-11-03-05, $L_{\mathrm{x}}=2.8 \times 10^{37} \mathrm{erg} \mathrm{s}^{-1}$ ): in the upper panel, PCA and HEXTE data points and corresponding best fit are reported; below: residuals for the best fit a), the fit excluding the absorption feature at $\sim 11 \mathrm{keV} \mathrm{b}$ ), and the one excluding the CRSF $\mathbf{c}$ ), respectively.

and faster changes in intensity are clearly detected in the $2 \mathrm{~s}$ resolution light curve.

The amplitude of change in count rate in both colours is more than twice in the decay $(\sim 0.15)$ compared to the flare phase $(\sim 0.05$, see Fig. 2$)$. Also, the colour values are higher larger during the flares, indicating a harder spectrum.

\subsubsection{Spectral analysis}

We fitted energy spectra with a continuum constituted by a photo-absorbed power law with a high-energy exponential cutoff, modified by a Gaussian line at $\sim 6.5 \mathrm{keV}$ with a fixed $0.5 \mathrm{keV}$ width to account for $\mathrm{Fe} \mathrm{K} \alpha$ fluorescence. A CRSF at an average central energy of $37 \mathrm{keV}$ was detected in high-flux observations only, above $L_{\mathrm{X}}=1.6 \times 10^{37} \mathrm{erg} \mathrm{s}^{-1}$, i.e. above $0.4 \times L_{\mathrm{Xmax}}$.

In addition, MXB 0656-072 shows significant residuals at $\sim 11 \mathrm{keV}$. They were fitted out by means of a Gaussian absorption-like profile, which allowed acceptable fits, passing from $\chi^{2} \sim 107$ for 70 DOF to $\chi^{2} \sim 85$ for 67 DOF, for a typical high-flux observation. This component is found at almost constant energy across the spectra, with a weighted mean value of $11.68 \pm 0.05 \mathrm{keV}$, and was consistently reported also by McBride et al. (2006) for the giant outburst and by Yan et al. (2012) for the normal ones. We found that this feature is only necessary in spectra where the CRSF is present as well. Its origin is uncertain.

Figure 3 shows a typical spectrum for a high-flux observation, with the corresponding residuals in the case of the best fit (a), the fit excluding the absorption at $\sim 11 \mathrm{keV}$ (b), and the one excluding the CRSF (c) from the model.

The best-fit main spectral parameters are shown in Fig. 4 as a function of the calculated 3-30 keV flux. Different marks and colours identify the two different phases of the outburst, the flaring and the smooth one. Points separated in time and 
E. Nespoli et al.: New insights into the Be/XRB system MXB 0656-072
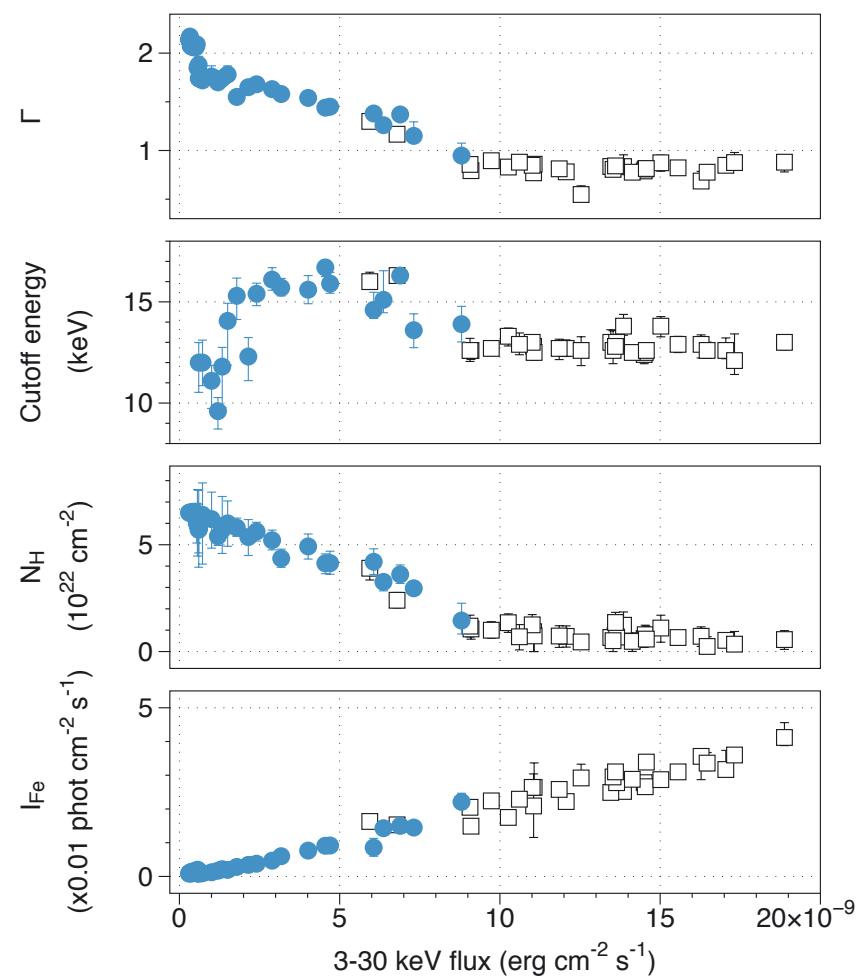

Fig. 4. Evolution of the main spectral parameters during the 2003 outburst. From the upper panel: photon index, cutoff energy, hydrogen column density, and Fe line intensity.

corresponding to different phases behave in a similar way, which only depends on flux. The power-law photon index decreases with X-ray flux, confirming the result from the colour analysis that as the X-ray flux increases the spectrum becomes harder (see also Fig. 2). The strength of the iron fluorescence line nicely correlates with flux, revealing an increase in the reprocessed material as the flux increases, as expected. The central energy of the $\mathrm{Fe}$ line remained fairly constant, with a mean value of $6.49 \pm 0.06 \mathrm{keV}$, and a mean equivalent width $(\mathrm{EW})$ of $0.34 \pm 0.07 \mathrm{keV}$.

The CRSF was almost constant during the outburst, with the following weighted mean values: $E_{\mathrm{c}}=36.8 \pm 0.4 \mathrm{keV}, \sigma=9.1 \pm$ 0.4 . These values are not compatible with the best-fit values by McBride et al. (2006), who found the CRSF at a central energy of $32.8_{-0.4}^{+0.5} \mathrm{keV}$. The discrepancy may be because McBride et al. (2006) obtained one spectral fit from a spectrum retrieved by summing up all the spectra from the flare phase of the outburst (from MJD 52932 to MJD 52964). In fact, when choosing four individual observations of that interval, the cyclotron line in the spectra of those four observations showed line energies above $33 \mathrm{keV}$ (see Fig. 3 in McBride et al. 2006). Our best-fit value for the central energy agrees with the one firstly reported by Heindl et al. (2003), of $E_{\mathrm{c}}=36 \pm 1 \mathrm{keV}$.

All the observed correlations, except for the iron line strength, are significant up to a "saturation" flux of $\sim 10^{-8} \mathrm{erg} \mathrm{cm}^{-2} \mathrm{~s}^{-1}$, which corresponds to $L_{\mathrm{X}}=$ $1.8 \times 10^{37} \mathrm{erg} \mathrm{s}^{-1}$. This luminosity roughly coincides with the large-amplitude flaring phase.

Because of the flaring activity, it is difficult to identify the peak of the outburst. The average $3-30 \mathrm{keV} \mathrm{X-ray} \mathrm{luminosity} \mathrm{in}$ the time interval MJD 52940-52970 is $L_{X}=2.5 \times 10^{37} \mathrm{erg} \mathrm{s}^{-1}$, although a maximum of $L_{\mathrm{X}}=3.7 \times 10^{37} \mathrm{erg} \mathrm{s}^{-1}$ was obtained
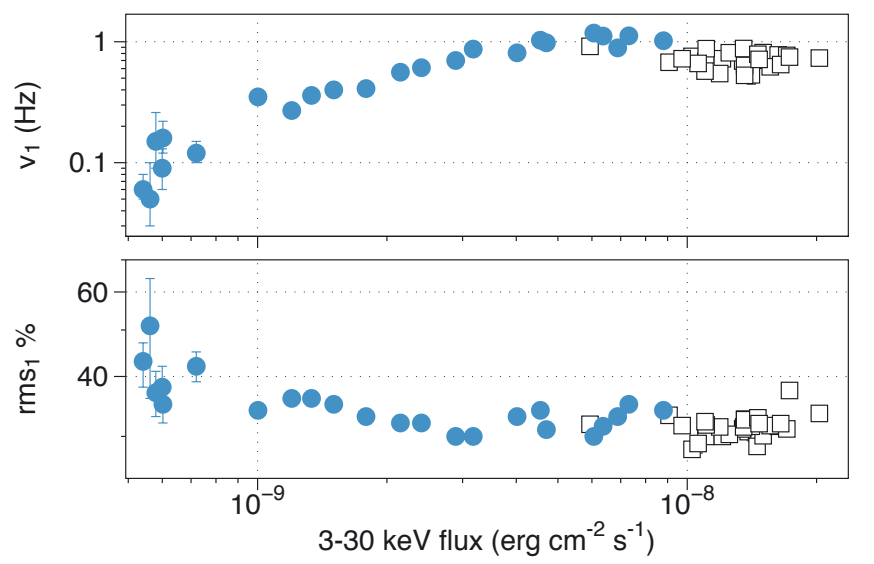

Fig. 5. Evolution of the timing parameters, characteristic frequency and fractional rms, of the best constrained noise component, $L_{1}$, during the 2003 outburst.

on MJD 52966.9. The lowest luminosity corresponds to the last point of the decay with $L_{\mathrm{X}}=1 \times 10^{36} \mathrm{erg} \mathrm{s}^{-1}$.

\subsubsection{Timing analysis}

In this work, we focus on the aperiodic variability of the system. For a study of the X-ray pulsations, see McBride et al. (2006). The neutron star spin frequency has a fundamental peak at $\sim 6 \mathrm{mHz}$, below our PSD frequency range $(0.008-32 \mathrm{~Hz})$. Thus, peaks derived from the neutron star's pulsations do not appreciably distort the continuum in the power spectra.

We fitted each PSD with the sum of Lorentzian functions with the objective of providing a unified phenomenological description of the timing behaviour of the system during the outburst. We denote each component as $L_{i}$, and its characteristic frequency $v_{\max }$ as $v_{i}$. According to the definition in Belloni et al. (2002), this is the frequency where the component contributes most of its variance per logarithmic frequency interval, $v_{\max }=\sqrt{v_{0}^{2}+(F W H M / 2)^{2}}$, where $v_{0}$ is the centroid frequency and FWHM is the full width at half maximum of the Lorentzian function. In this work, we always refer to characteristic frequencies $v_{\max }$.

The low and middle-frequency noise $\left(L_{0}\right.$ and $\left.L_{1}\right)$ is accounted for by zero-centred Lorentzians with a characteristic frequency that is generally lower for low-flux pointings, and higher for high-flux observations. The corresponding fractional rms varies during the outburst, in anti-correlation with flux. These components are the only ones required during all the smooth decay phase (after MJD 52 975) and part of the flaring phase of the outburst, up to $f_{x} \approx 1 \times 10^{-8} \mathrm{erg} \mathrm{cm}^{-2} \mathrm{~s}^{-1}$. Beyond that luminosity, an additional component is necessary, $L_{2}$, whose characteristic frequency varies in the range $\sim 1-5 \mathrm{~Hz}$, whereas the fractional amplitude of variability varies between $10 \%$ and $20 \%$, without a clear dependence on flux. Unlike $L_{0}$ and $L_{1}$, this component has, in general, a non-zero centroid frequency, and its average value for the $Q$-factor, defined as $Q=v_{0} / F W H M$, is $\sim 0.6$, denoting a narrower feature compared to $L_{0}$ and $L_{1}$. Figure 5 presents the evolution of the characteristic frequency and rms over the outburst for the $L_{1}$ component, the best constrained one. Up to $f_{x} \approx 1 \times 10^{-8} \mathrm{erg} \mathrm{cm}^{-2} \mathrm{~s}^{-1}$, as the flux increases the characteristic frequency increases, whilst after that luminosity it remains approximately constant. The rms variability shows an opposite trend, decreasing as the flux increases, and then saturating. Figure 6 shows the relation between the $L_{1}$ and $L_{2}$ characteristic 


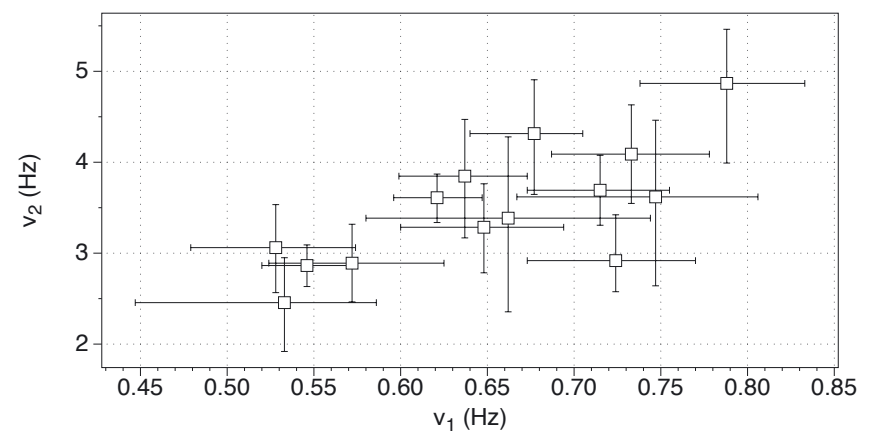

Fig. 6. Relation between the maximum frequencies of the $L_{1}$ and $L_{2}$ components.

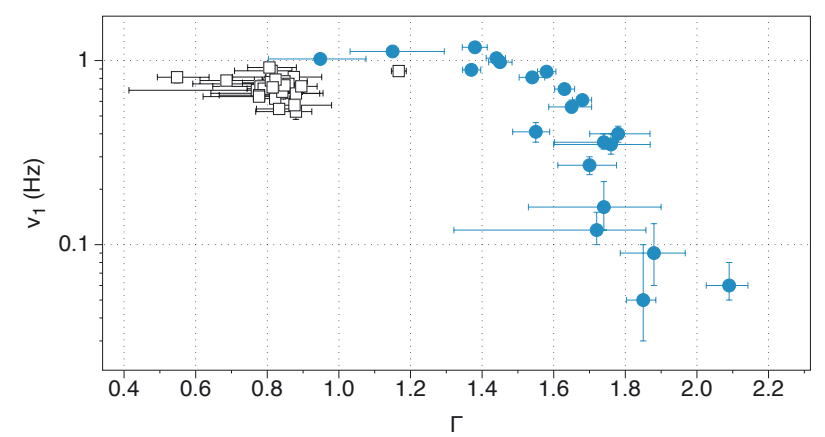

Fig. 7. Relation between the photon index and the characteristic frequency of the $L_{1}$ broad-noise component.

frequencies; although within some scattering, the two frequencies follow a correlated trend in all their range of variation.

We studied the correlated spectral/timing behaviour, and found that during the very last part of the flaring phase, and the whole decay, the photon index $\Gamma$ and the central frequency of the main timing component, $v_{1}$, vary in an anti-correlated way (Fig. 7). During the flares the $L_{1}$ frequency shows only slight variation due to the appearance of $L_{2}$, so that no spectral/timing relation could be expected.

\subsection{Type I outbursts}

In addition to the major (type II) outburst reported in previous sections, MXB 0656-072 underwent a series of four fainter outbursts between November 2007 and November 2008. The PCA began to monitor these outbursts at the end of the first one. All outbursts exhibited similar peak luminosities $\left(L_{\text {peak }}=\right.$ $\left.1.2 \times 10^{37} \mathrm{erg} \mathrm{s}^{-1}\right)$. This luminosity is about three times lower than that of the November 2003 type-II outburst.

We show in Fig. 8 the simultaneous $\mathrm{H} \alpha$ measurements and ASM light curve. The outbursts are separated by $\sim 100$ days and in between the major peaks, other minor peaks are observed. The onset of these outbursts coincided with an optical maximum brightness of the donor: at around MJD 54500 (February 2008) the equivalent width of the $\mathrm{H} \alpha$ line seems to have reached a maximum value of $\sim 25 \AA$ (Yan et al. 2012).

In our X-ray spectral analysis, in order to best constrain the CRSF, hardly detectable at very low flux, we extracted three average spectra in three ranges of luminosities and fitted them between 3-60 keV. The model employed was the same as for type-II outburst, and all the components were needed to obtain acceptable fits at in all the three spectra. Spectral parameters are shown in Table 3. Similarly to our analysis of the giant outburst, we also generated one energy spectrum for each observing

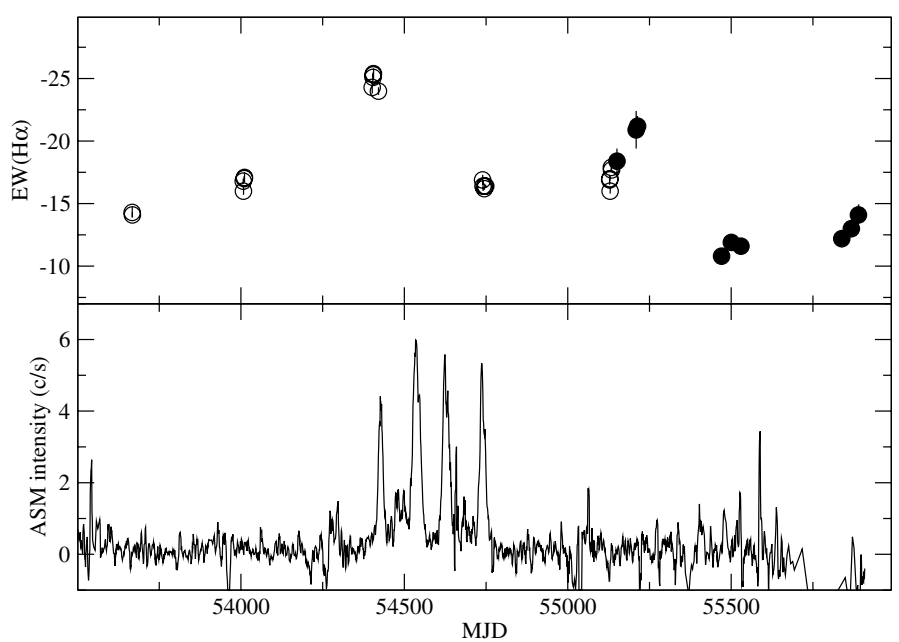

Fig. 8. ASM light curve and $\mathrm{H} \alpha$ equivalent width evolution. A 5-day running average was applied to the 1-day ASM light curve to reduce the noise. Open circles correspond to observations by Yan et al. (2012) and filled circles to our observations (see Table 2).

interval and studied the evolution of the spectral parameters. We fixed the CRSF parameters in each single spectrum to the ones obtained from the corresponding flux average spectrum, and fitted each one between $3 \mathrm{keV}$ and $30 \mathrm{keV}$. We found that the spectral parameters follow similar trends to those seen during the type II outburst (Fig. 4). The photon index decreases as the flux increases, as in the type II outburst, although with a steeper dependence with flux. The iron line energy does not significantly vary, while its intensity, expressed as normalisation, tightly correlates with luminosity. The iron line width was fixed at $0.5 \mathrm{keV}$. The hydrogen column density generally anti-correlates with flux, while the cutoff energy does not show any smooth relation with luminosity, although it displays lower values at higher flux and vice versa.

\section{Discussion}

We have performed a detailed X-ray and optical analysis of the poorly studied hard X-ray transient MXB 0656-072. All the available observational data indicate that MXB 0656-072 is a member of the class of massive X-ray binaries known as $\mathrm{Be} / \mathrm{X}$-ray binaries. X-rays are produced in the vicinity of the compact object, while the optical variability comes from the young and massive companion. The detection of X-ray pulsations, the transient nature of the X-ray emission, and the characteristics of the X-ray spectrum (power-law continuum modified by an exponential cutoff and the presence of fluorescence iron line and cyclotron feature) are typical of neutron star binaries. The observation of Balmer lines in emission favours the Be/XRB classification. Furthermore, the long-term X-ray variability, consisting of giant (type II) and minor recurrent outbursts (type I) are typical of $\mathrm{Be} / \mathrm{XRB}$.

\subsection{Optical observations}

The optical counterpart to MXB 0656-072 was classified as a $09.5 \mathrm{Ve}$ star, refining previous classification by Pakull et al. (2003). Its spectrum is strongly affected by emission, with the first three lines of the Balmer series $(\mathrm{H} \alpha, \mathrm{H} \beta$, and $\mathrm{H} \gamma)$ showing an emission profile, while the next two $(\mathrm{H} \delta$ and $\mathrm{H} \epsilon)$ are partially filled in with emission. This extra emission is thought to 
Table 3. Type-I outburst spectral analysis for average spectra at different flux ranges.

\begin{tabular}{lccc}
\hline \hline Spectral parameter & Low flux $^{a}$ & Med. flux $^{b}$ & High flux $^{c}$ \\
\hline$\Gamma$ & $1.08 \pm 0.08$ & $0.83 \pm 0.04$ & $0.49 \pm 0.03$ \\
Cutoff en. $(\mathrm{keV})$ & $16.9_{-2.3}^{+2.5}$ & $14.4_{-0.5}^{+0.9}$ & $10.5 \pm 0.02$ \\
Pow. norm. $\left(\mathrm{ph} / \mathrm{keV} / \mathrm{cm}^{2} / \mathrm{s}\right)$ & $0.11_{-0.02}^{+0.04}$ & $0.12 \pm 0.01$ & $0.115_{-0.007}^{+0.004}$ \\
$N_{\mathrm{H}}\left(10^{22} \mathrm{~cm}^{-2}\right)$ & $4.1 \pm 0.5$ & $2.8 \pm 0.3$ & $1.9 \pm 0.3$ \\
$E_{\mathrm{Fe}}(\mathrm{keV})$ & $6.5 \pm 0.1$ & $6.45_{-0.04}^{+0.05}$ & $6.5 \pm 0.4$ \\
$E W_{\mathrm{Fe}}$ & $0.06 \pm 0.02$ & $0.14_{-0.8}^{+0.2}$ & $0.17_{-0.11}^{+0.22}$ \\
$E_{10 \mathrm{keV}-\text { Gaus }}$ & $10.3_{-0.5}^{+0.4}$ & $10.6_{-0.1}^{+0.3}$ & $10.6_{-0.1}^{+0.2}$ \\
$\sigma_{10 \mathrm{keV}-\text { Gaus }}$ & $6.5_{-0.6}^{+0.9}$ & $4.2_{-0.5}^{+0.3}$ & $2.9_{-0.23}^{+0.24}$ \\
$\tau_{10 \mathrm{keV}-\text { Gaus }}$ & $15.8_{-3.9}^{+6.2}$ & $2.3_{-0.5}^{+0.4}$ & $1.05_{-0.12}^{+0.16}$ \\
$E_{\mathrm{cyc}}$ & $31.9_{-2.7}^{+1.3}$ & $35.6_{-1.3}^{+2.1}$ & $35.0_{-1.0}^{+1.7}$ \\
$\sigma_{\mathrm{cyc}}$ & $11.2_{-0.9}^{+3.2}$ & $8.5_{-1.2}^{+1.4}$ & $8.8_{-1.3}^{+2.2}$ \\
$\tau_{\mathrm{cyc}}$ & $58.0_{-8.9}^{+13.2}$ & $14.0_{-3.7}^{+8.1}$ & $13.0_{-3.8}^{+7.8}$ \\
\hline
\end{tabular}

Notes. ${ }^{(a)}<10^{-9} \mathrm{erg} \mathrm{cm}^{-2} \mathrm{~s}^{-1}{ }^{(b)}(1-2.5) \times 10^{-9} \mathrm{erg} \mathrm{cm}^{-2} \mathrm{~s}^{-1}{ }^{(c)}>2.5 \times$ $10^{-9} \mathrm{erg} \mathrm{cm}^{-2} \mathrm{~s}^{-1}$.

arise from the equatorial disc around the Be star. The picture described by optical spectroscopy is fully consistent with the so-called "Be-phenomenon" and confirms that the system is a $\mathrm{Be} / \mathrm{XRB}$.

From the analysis of type-I outbursts, Yan et al. (2012) find an orbital period of $101.2 \mathrm{~d}$. Once the period is known, we can use two important relationships involving the orbital period of the system, the $P_{\text {spin }}-P_{\text {orb }}$ (Corbet 1986) and $P_{\text {orb }}-E W(\mathrm{H} \alpha)$ (Reig et al. 1997; Reig 2011) diagrams, to support the orbital period found. In the first diagram (see Fig. 6 in Yan et al. 2012), the source is clearly located in the region occupied by $\mathrm{Be} / \mathrm{XRBs}$; in the second one (Fig. 9), a $\sim 100$ d orbital period fits nicely in the expected $\mathrm{EW}(\mathrm{H} \alpha)$ vs. orbital period relation. The $P_{\text {orb }}-E W(\mathrm{H} \alpha)$ correlation is a consequence of tidal truncation of the Be star's circumstellar disc by the neutron star. Assuming that the equivalent width of the $\mathrm{H} \alpha$ line, $\mathrm{EW}(\mathrm{H} \alpha)$, provides a good measure of the size of the circumstellar disc (Quirrenbach et al. 1997; Tycner et al. 2005), the $P_{\text {orb }}-E W(\mathrm{H} \alpha)$ correlation indicates that systems with long orbital periods have larger discs, while narrow orbit systems contain smaller discs. In short orbital period systems, the neutron star prevents the formation of extended discs. As can be seen in Fig. 9, an orbital period of $\sim 100$ days agrees very well with the maximum $\operatorname{EW}(\mathrm{H} \alpha)$ of $\sim-25 \AA$.

\subsection{Type-II outburst}

The profile of the type-II outburst in MXB 0656-072 is unusual among Be/XRB. Although flaring behaviour has been seen in other systems (e.g., EXO 2030+375, A0535+26, Klochkov et al. 2011; Caballero et al. 2008), the profile of the outbursts tends to be more symmetric and to have a smoother peak. In MXB 0656072, the flaring activity covers a substantial part of the outburst (Fig. 2). It was found that magneto-hydrodynamic instabilities at the inner edge of the accretion disc may produce oscillations in the accreting flow, possibly leading to the observed behaviour (Apparao 1991; Postnov et al. 2008; D’Angelo \& Spruit 2010). Especially, D'Angelo \& Spruit (2010) show that, if the accretion disc is truncated by the neutron star's magnetic field outside but close to the corotation radius (at which the Keplerian frequency in the disc equals the star's rotational frequency), then

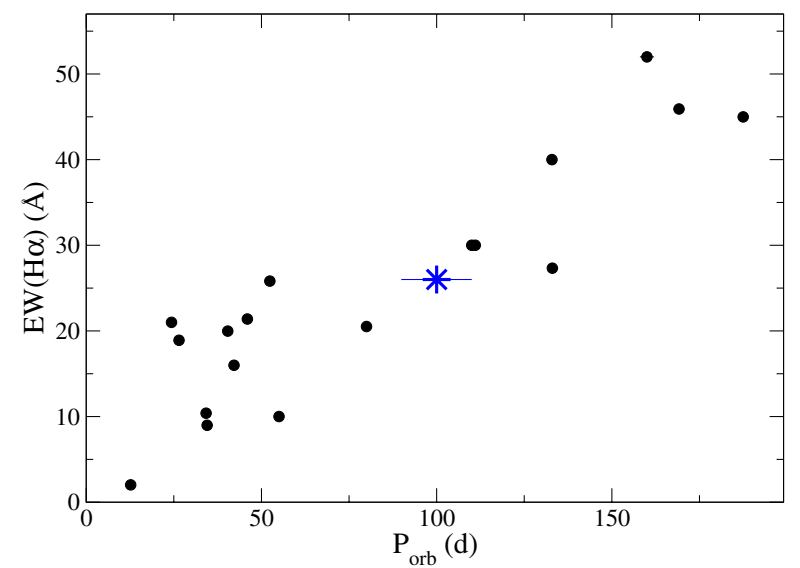

Fig. 9. $P_{\text {orb }}-E W(\mathrm{H} \alpha)$ diagram. The star symbol marks the position of MXB 0656-072.

the accretion becomes time-dependent and takes the form of repeated bursts.

We found correlated behaviour of both the SC and the HC with flux, which corresponds to a general hardening of the spectra as the flux increases. The dependence of the SC on luminosity does, in appearance, contrast with the observed negative correlation in other sources. Reig (2008) analysed the spectral and timing properties of four $\mathrm{Be} / \mathrm{XRBs}$ during giant outburst, identifying two source states, a low-luminosity horizontal branch (HB) and a high-luminosity diagonal branch (DB). During the outburst, the sources spend most of the time in the DB, during which the SC anti-correlates with flux. The transition to the HB inverts this trend, and the SC starts correlates with it. The correlation between the SC and luminosity observed in MXB 0656072 thus reveals that the source never undergoes the transition to the $\mathrm{DB}$, but remains in the $\mathrm{HB}$ during all the duration of the outburst.

The hardening of the spectrum at high flux is confirmed by spectral analysis: from simultaneous spectral fitting of PCA and HEXTE spectra, we found a clear anti-correlated behaviour of the spectral index with flux (Fig. 4) up to flux $\sim 1.2 \times$ $10^{-8} \mathrm{erg} \mathrm{cm}^{-2} \mathrm{~s}^{-1}$, or $L_{\mathrm{X}}=2.3 \times 10^{37} \mathrm{erg} \mathrm{s}^{-1}$, when the trend flattens. The spectral hardening with increasing flux can be interpreted with simple Comptonisation models: the X-ray emission from HMXBs is thought to arise in an accretion column, close to the neutron star's surface, as a result of Comptonisation of soft photons injected in the accretion flow from the NS thermal mound, by high-energy electrons of the accreting matter (Becker $\&$ Wolff 2007). Increasing X-ray luminosity would mainly correspond to increasing mass accretion rate, which can be translated into averagely increasing number of up-scattering collisions of photons with electrons, resulting in a harder spectrum.

After 1A 1118-615 (Nespoli \& Reig 2011), MXB 0656-072 is the second X-ray pulsar to show correlated spectral/aperiodic behaviour (Fig. 7). This translates into a necessary connection between the two physical regions responsible for producing the two phenomenologies, the accretion column on one side, where energy spectra arise, and the accretion disc on the other side where, according to the "perturbation propagation" model, the aperiodic variability is located (Revnivtsev et al. 2009, and references therein). In this model, in fact, the X-ray variability is caused by perturbations in the inner disc flow, at different radii. The two regions are physically separated, being the first close to the NS polar cap regions, and the second confined outside the magnetosphere, but our results on two systems show that they are somehow coupled. 
In general, the X-ray variability of MXB 0656-072 during the type-II outburst resembles that of 1A 1118-615 at many levels. The colours behave exactly the same in the two sources (both correlate with flux). The spectral parameters have the same trend in the two sources, and in both cases the relation with flux ceases at some saturation luminosity. In MXB 0656-072 this saturation is reached at $0.5 \times L_{\mathrm{X} \max }$, while in $1 \mathrm{~A} 1118-615$ at $0.7 \times L_{\mathrm{Xmax}}$. Finally, the correlations between the two broadband timing components and between spectral and timing parameters are also observed in both sources, making them very similar during a type-II outburst, although no flaring activity is observed in $1 \mathrm{~A}$ 1118-615.

A peculiar feature in the X-ray energy spectra of MXB 0656072 is an absorption-line-like profile at an average constant energy between 11-12 keV. This component was investigated by Coburn (2001), who detected it in the range 8-12 keV in the spectra of many X-ray pulsars. The feature was consistently observed at the same energy, irrespective the CRSF energy, and moreover, it was evident in some systems that do not display a cyclotron line. This made Coburn (2001) conclude that the component should not be a magnetic effect. The feature seems to be intrinsic to X-ray pulsars spectra, since it was observed with different instruments (besides RXTE, Ginga and BeppoSAX, Mihara 1995; Santangelo et al. 1998). In the case of MXB 0656072, this component is only found in spectra where the CRSF is present as well, although no other relation could be established between the two features.

\section{Conclusions}

We presented a detailed X-ray and optical study of MXB 0656072 covering both types of $\mathrm{X}$-ray variability observed in a $\mathrm{Be} / \mathrm{XRB}$, namely type-I and type-II outbursts. The major outburst is characterised by flare-like behaviour during its peak, followed by smoother decay. We interpreted the flaring activity as possibly due to magneto-hydrodynamic instabilities at the inner edge of the accretion disc. The colour and spectral analyses reveal a hardening of the spectra as the luminosity increases, which can be understood in the framework of the models for spectra production in X-ray pulsars. The analysis of aperiodic variability shows correlated behaviour of the timing parameters with flux, which translates into a correlation between spectral/timing features and can be interpreted as an interconnection between the two physical regions responsible for the two phenomenologies. All the X-ray behaviour during the type-II outburst resembles that of 1A 1118-615, although no such flaring activity was observed in that system. The spin period vs. $\mathrm{EW}(\mathrm{H} \alpha)$ relation confirmed the orbital period proposed for the source. The full multiwavelength analysis corroborates the $\mathrm{Be} / \mathrm{XRB}$ nature of the system.

Further observations during major outbursts are needed in order to explore the nature of the flaring activity and the timing/spectral correlation detected in this work. Deeper comprehension of the interaction between the magnetosphere and the accretion disc is also necessary to explain the correlated behaviour of the spectral and aperiodic features.

Acknowledgements. E.N. acknowledges a "VALi+d" postdoctoral grant from the "Generalitat Valenciana" and was supported by the Spanish Ministry of Economy and Competitiveness under contract AYA 2010-18352. P.R. acknowledges support by the Programa Nacional de Movilidad de Recursos Humanos de Investigación 2011 del Plan Nacional de I-D+i 2008-2011 of the Spanish Ministry of Education, Culture and Sport. P.R. also acknowledges partial support by the COST Action ECOST-STSM-MP0905-020112-013371. ASM quick-look results provided by the ASM/RXTE team.

\section{References}

Apparao, K. M. V. 1991, ApJ, 375, 701

Arnaud, K. A. 1996, in Astronomical Data Analysis Software and Systems V, eds. G. H. Jacoby, \& J. Barnes, ASP Conf. Ser., 101, 17

Becker, P. A., \& Wolff, M. T. 2007, ApJ, 654, 435

Belloni, T., \& Hasinger, G. 1990, A\&A, 230, 103

Belloni, T., Psaltis, D., \& van der Klis, M. 2002, ApJ, 572, 392

Bradt, H. V., Rothschild, R. E., \& Swank, J. H. 1993, A\&AS, 97, 355

Caballero, I., Santangelo, A., Kretschmar, P., et al. 2008, A\&A, 480, L17

Clark, G. W., Schmidt, G. D., \& Angel, J. R. P. 1975, IAU Circ., 2843, 1

Coburn, W. 2001, Ph.D. Thesis, University of California, San Diego

Corbet, R. H. D. 1986, MNRAS, 220, 1047

D’Angelo, C. R., \& Spruit, H. C. 2010, MNRAS, 406, 1208

Ekström, S., Meynet, G., Maeder, A., \& Barblan, F. 2008, A\&A, 478, 467

Hayasaki, K., \& Okazaki, A. T. 2004, MNRAS, 350, 971

Heindl, W., Coburn, W., Kreykenbohm, I., \& Wilms, J. 2003, The Astronomer's Telegram, 200, 1

Kaluzienski, L. J. 1976, IAU Circ., 2935, 5

Klochkov, D., Ferrigno, C., Santangelo, A., et al. 2011, A\&A, 536, L8

Landolt, A. U. 2009, AJ, 137, 4186

McBride, V. A., Wilms, J., Coe, M. J., et al. 2006, A\&A, 451, 267

Mihara, T. 1995, Ph.D. Thesis, Dept. of Physics, Univ. of Tokyo (M95)

Miyamoto, S., Kimura, K., Kitamoto, S., Dotani, T., \& Ebisawa, K. 1991, ApJ, 383,784

Morgan, E., Remillard, R., \& Swank, J. 2003, The Astronomer's Telegram, 199, 1

Motch, C., Stella, L., Janot-Pacheco, E., \& Mouchet, M. 1991, ApJ, 369, 490

Nespoli, E., \& Reig, P. 2011, A\&A, 526, A7

Nowak, M. A., Vaughan, B. A., Wilms, J., Dove, J. B., \& Begelman, M. C. 1999, ApJ, 510, 874

Pakull, M. W., Motch, C., \& Negueruela, I. 2003, The Astronomer's Telegram, 202,1

Porter, J. M., \& Rivinius, T. 2003, PASP, 115, 1153

Postnov, K., Staubert, R., Santangelo, A., et al. 2008, A\&A, 480, L21

Quirrenbach, A., Bjorkman, K. S., Bjorkman, J. E., et al. 1997, ApJ, 479, 477

Reig, P. 2008, A\&A, 489, 725

Reig, P. 2011, Ap\&SS, 332, 1

Reig, P., Fabregat, J., \& Coe, M. J. 1997, A\&A, 322, 193

Revnivtsev, M., Churazov, E., Postnov, K., \& Tsygankov, S. 2009, A\&A, 507, 1211

Santangelo, A., del Sordo, S., Segreto, A., et al. 1998, A\&A, 340, L55

Tycner, C., Lester, J. B., Hajian, A. R., et al. 2005, ApJ, 624, 359

Walborn, N. R. 1971, ApJS, 23, 257

Walborn, N. R., \& Fitzpatrick, E. L. 1990, PASP, 102, 379

Yan, J., Zurita Heras, J. A., Chaty, S., Li, H., \& Liu, Q. 2012, ApJ, 753, 73 